\title{
Estimation of platelet adhesiveness on whole blood and platelet-rich plasma
}

\author{
SIDNEY SHAW, GEOFFREY D. PEGRUM, AND SYLVIA WOLFF \\ From the Department of Haematology, Charing Cross Hospital Medical School, London
}

SYNOPSIS Platelet adhesive tests were carried out in parallel on whole blood and platelet-ric $\overrightarrow{\mathrm{c}}$ plasma. A column of glass microspherules was used and adenosine diphosphate (ADP) w⿳⺈冂大 added to the platelet-rich plasma before passage through the column.

Preliminary observations were made to show the effect of the containers and the antion coagulant used. The influence on platelets when stored at $4^{\circ} \mathrm{C}$ and standing or mixing at room temperature was also assessed. The apparatus used to measure adhesiveness was designed produce a constant rate of flow through a standard column of glass microspherules.

Using both methods, adhesiveness was studied on 70 healthy blood donors. The resuff

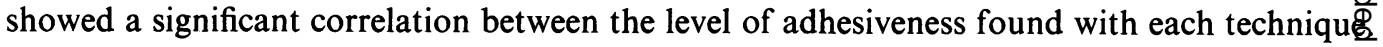

One of the main difficulties in performing platelet adhesive studies in vitro is that different methods may not measure the same aspect of adhesiveness (O'Brien, 1966 and 1969); also the degree of relevance of these measurements to events in vivo is unknown. O'Brien has stated that measurement of adhesiveness can only be considered in the context of what has been found by a given investigator in a particular laboratory on a specific day; he has also shown (1967) that platelets from one patient may be more affected by one aggregating agent than another. Furthermore, different workers using similar techniques have been unable to obtain reproducible results.

Changes in platelet adhesiveness recorded by a certain technique in a given clinical situation may, if the method is not rigidly standardized, have a false significance. Markedly different results have been found in certain disorders, such as von Willebrand's disease where ADP aggregation is normal; glass adhesiveness appears defective on rapid passage through a column of glass beads but slowing the rate of passage produces similar results as on normal controls (O'Brien and Heywood, 1967). It is therefore essential to standardize the technique used and to have adequate controls on healthy subjects before Received for publication 13 May 1969. using, and interpreting, results in various disease processes. Many of the experimental conditions which influence adhesiveness were initially studieg by Hellem (1960a). Since that time many other factors have been shown to affect platelet adhe. sion. These factors fall into two main groups: of the one hand, those which affect the test conde tions and blood sample, such as the time interva following blood taking (Fyfe and Hamilton 1967), the time interval after adding ADP (Bloo and Davies, 1967), alterations in the packed cel volume of red cells (Hellem, 1960b), the duration of centrifugation before the tests on platelet-rict plasma (McBride, 1968). The second group ar:्E those conditions which alter the milieu in the patient such as smoking (Murchison and Fyfe 1966), ingestion of glucose (Bridges, Dalbs Millar, and Weaver, 1965), fat (McDonald an' Edgill, 1958), taking aspirin (O'Brien, 1968) and other drugs, and exercise (Ikkala, Myllylä, an Sarajas, 1966).

The object of the present study was to see if $\frac{\vec{B}}{\mathrm{~B}}$ was feasible, after standardizing two methods of platelet adhesiveness, to obtain comparable results. The procedures chosen both involveg platelet adhesion to glass beads, the first using. whole blood and the second using citrated plasma with ADP followed by adherence to glass beads. 
These tests were undertaken in 70 normal subjects, both techniques being carried out on blood samples taken at the same time. Before the study, each step of both techniques was examined carefully and the factors which might cause variable results were sought, and, as far as possible, standardized. The preliminary findings of the factors influencing the experimental systems from which the two procedures were developed will be presented first, and, then using both methods, the results on healthy donors will be compared.

\section{Materials and Methods}

Venous blood was obtained from 70 healthy blood donors between the ages of 19 and 70 . In each case the blood was taken in the morning before lunch but at a variable time following breakfast. We were unable to control the amount of food eaten, smoking, or the amount of exercise taken before the tests, and these factors could all possibly influence the results.

Blood samples were collected using a disposable plastic syringe and disposable needle and placed in either plastic containers or siliconized glass tubes. Each tube contained $3 \cdot 13 \%$ sodium citrate as anticoagulant. Initially, a 1 in 10 dilution was used, but later a 1 in 12 dilution was preferred as this was shown to have less effect on the platelets. After being added, the blood was carefully mixed with the citrate by gentle inversion 15 times; this method of mixing was used throughout.

Glass microspherules of a diameter between $0 \cdot 102$ and $0 \cdot 124 \mathrm{~mm}$ were prepared in the following way. They were first washed well with $20 \% \mathrm{HCl}$, rinsed approximately 20 times in water, distilled water being used on the last four occasions; the microspherules were then dried in a hot air oven before use.

Adenosine diphosphate was prepared in a final concentration of $50 \mu \mathrm{g} / \mathrm{ml}$. This was made up freshly once a month and stored in small aliquots at minus $20^{\circ} \mathrm{C}$. It was brought to room temperature 30 minutes before use, and not refrozen. After suitable dilution, platelets were counted using a Coulter counter model F. Counts were always performed in duplicate or triplicate and an agreement of within $5 \%$ was accepted as satisfactory, otherwise the count was repeated. The adhesive platelet count was the difference in the number of platelets counted before and after the procedure expressed as a percentage. Polystyrene containers of uniform size $(6 \mathrm{~cm} \times$ $2 \mathrm{~cm}$ ) were used throughout except in the experiments with siliconized tubes which were $11 \mathrm{~cm} \times$ $1.4 \mathrm{~cm}$ and coated with silicone (MS 1107).

\section{Preliminary Procedures}

These investigations were carried out using blood samples from healthy donors.
MIXING OF BLOOD SPECIMENS

It was found that the platelet count fell when citrated whole blood or platelet-rich plasma was allowed to rotate at $28 \mathrm{rpm}$ on a Matburn wheel for any length of time; the reduction in platelets was most marked in the polystyrene containers but also occurred in the siliconized tubes. Presumably, mixing of the specimens initiates the process of adhesion. Eastham (1964) has previously shown that platelets adhere to polystyrene surfaces, and therefore the minimum agitation of the specimens before testing is essential.

EFFECT ON PLATELET COUNT OF KEEPING BLOOD SPECIMENS AT $4^{\circ} \mathrm{C}$

Blood samples $(3 \mathrm{ml})$ were placed in polystyrene tubes with citrate as anticoagulant at $4^{\circ} \mathrm{C}$ for 24 hours without rotation and the platelet count was compared with the initial count. In doing this experiment we found that there was an optimum time for satisfactory mixing of blood to obtain a uniform platelet count. After mixing on a circular wheel at $28 \mathrm{rpm}$ for three minutes and five minutes the platelet counts showed irregular results in repeated tests. Those mixed for 10 minutes gave a more uniform level. Thereafter, platelet counts performed on blood samples which had been left standing were rotated for 10 minutes at $28 \mathrm{rpm}$ before the specimen was taken for testing or platelet counting. With suitable mixing, blood which was left overnight at $4^{\circ} \mathrm{C}$ was found to have no significant drop in platelet count in subsequent counts. Platelet counts after mixing for 10 minutes at $28 \mathrm{rpm}$ showed a closer agreement using siliconized glass containers than with polystyrene tubes, but the difference was slight.

EFFECT ON PLATELET COUNT OF LEAVING BLOOD AT ROOM TEMPERATURE

Blood was taken into polystyrene tubes and siliconized glass tubes with citrate as an anticoagulant, left at room temperature for periods of up to three hours, and mixed by rotation 10 minutes before carrying out the platelet count. The results in 10 repeated tests using polystyrene tubes and siliconized tubes showed no significant fall in platelet counts although using siliconized tubes gave marginally more accurate results. The same experiment was repeated using platelet-rich plasma; once again there was little difference in the platelet count in polystyrene or siliconized containers.

\section{ANTICOAGULANT}

We also experimented with the dilution of sodium citrate and used buffered citrate as well as $3 \cdot 13 \%$ sodium citrate. It was found that with 1 in 12 dilutions, which we used in all subsequent experiments, the platelet count had a greater stability after standing at room temperature than when in 
a 1 in 10 dilution. Buffered citrate was not found to offer any advantage.

From these preliminary experiments we confirmed that the results were variable if conditions were not rigidly standardized, especially with regard to the anticoagulant used, the size of the container, the type of the container, and the amount of agitation of the sample. Although siliconized containers were slightly superior to polystyrene containers, it was found more practical to use polystyrene tubes, taking care to keep a constant experimental system in each technique and to avoid over-mixing the samples.

\section{Technique of Platelet Adhesiveness}

It was decided to use a column of glass microspherules for these studies and to carry out all procedures at room temperature.

The spherules were put inside a plastic syringe with an internal diameter of $4 \mathrm{~mm}$ to give a column $6.5 \mathrm{~cm}$ long. The wide end was fitted with a plastic adaptor and tubing, the other end was fitted to a $5 \mathrm{ml}$ syringe in which the blood or platelet-rich plasma was placed. The glass beads were prevented from leaving the syringe by a small plug of siliconized glass wool. The connecting plastic tubing had a diameter of $4 \mathrm{~mm}$ and was as short as possible. The column of microspherules was fixed vertically and the $5 \mathrm{ml}$ syringe horizontally. A simple mechanical hand-turned system of pushing the blood through the column was preferred to using an electric motor, which has been used in previous studies. With an automatic system there is a possibility that if partial obstruction of the flow occurs the rate through the column will change without the experimenter being aware of this change. Using a thread of known size which gives an exact movement in a given time, any change in pressure would immediately be apparent to the operator and the test can be repeated. This procedure produces a uniform flow and in many repetitive tests in our hands it was found to be precise. The exact whole blood technique was to place $2 \mathrm{ml}$ blood, after taking a sample for the initial platelet count, into a $5 \mathrm{ml}$ syringe. This was then attached to the apparatus and the sample pushed through the column of glass microspherules at the rate of $1 \mathrm{ml}$ in 7 seconds; the procedure takes 14 seconds, and, at exactly 20 seconds after the start, the sample which had passed through the column and collected in a plastic tube was mixed gently for 20 seconds and a second platelet count carried out.

The modification of the technique was as follows: platelet-rich plasma was obtained by centrifuging the citrated blood at $1,500 \mathrm{rpm}$ for 15 minutes and $0.5 \mu \mathrm{g}$ of ADP was added to $1 \mathrm{ml}$ of plasma after taking a sample for the initial platelet count. After the addition of ADP the specimen was mixed 10 times by gentle inversion and then
$2 \mathrm{ml}$ drawn up into the $5 \mathrm{ml}$ syringe. Exactly one minute after adding the ADP the sample was pushed through the column in the manner de⿻ scribed and the plasma was collected in poly styrene tubes containing $2.4 \mathrm{mg}$ of ethylene diamine tetracetic acid (EDTA). Twenty? seconds after the commencement of passage through the column, the sample collected was mixed gently for 20 seconds and a platelet coun performed. Preliminary experiments were neces $\Phi$ sary to ascertain the quantity of glass microa spherules to be used and also the amount of ADP to be added to the platelet-rich plasma.

After carrying out several experiments with varying quantities of glass microspherules it waळ decided to use $1.5 \mathrm{~g}$ for both citrated whole blood and citrated platelet-rich plasma. Thesiu quantities produced a level of platelet adhesive $e_{\tilde{N}}^{\omega}$ ness of about $50 \%$ using healthy donors.

A series of experiments was then performed to demonstrate the changes in adhesiveness whiclo occurred at various time intervals.

\section{Results}

The variation of platelet adhesiveness over 240 minutes using whole blood is shown in Figure 1 웅 The upper and lower lines indicate the range of results in five tests. After an initial fall in th samples taken 10 minutes after venesection, ther was a gradual rise in adhesiveness in the sample taken up to 60 minutes. From 60 to 120 minute? the level was remarkably constant, following which there was a slight increase in adhesivenes in samples left up to 240 minutes.

The variation in platelet adhesiveness ove 240 minutes using citrated plasma is shown Figure 2. The upper and lower lines indicate the range of results in four tests. Once again there wa@ an initial fall in the level of adhesiveness witk blood taken 10 to 20 minutes previously, followe $\Phi$ by a rise with the samples taken up to 60 minutess The levels reached between 60 and 120 minutes. were fairly constant, although not as close as in the whole blood results. Afterwards there was a gradual rise with plasma left for 180 minutes and an apparent slight fall up to 240 minutes

On this evidence it was decided to measure the adhesiveness between one and two hours followe ing venesection. At this time interval the most stable results were achieved and this interval also allowed sufficient time to collect the sampled and transmit them to the laboratory. This stabilito was tested using 16 healthy donors and estima ing adhesiveness at one and two hours afte venesection. With whole citrated blood the average adhesiveness was $55 \%$ at one hour and $54 \%$ at two hours with a variation of $40 \%$ to $71 \frac{\%}{6}$ at one hour and 35 to $72 \%$ at two hours. On 10 . tests at one and two hours using citrated plasmo the mean adhesive levels were $45 \%$ and $43 \%$ 


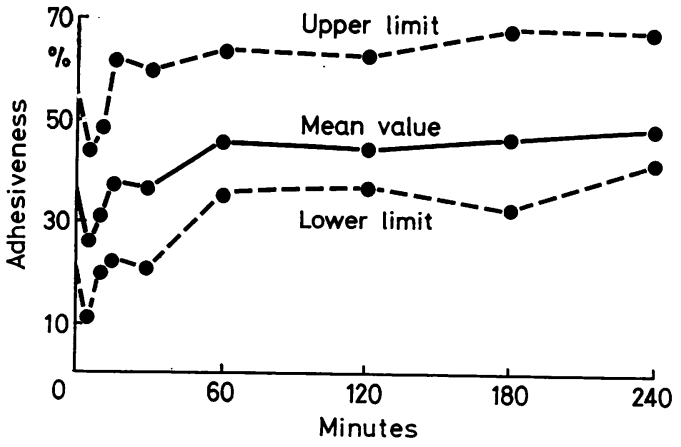

Fig. 1 Variation in platelet adhesiveness using whole blood.

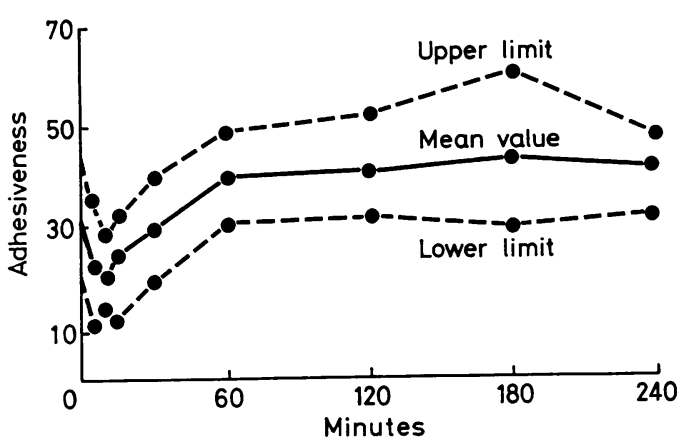

Fig. 2 Variation in platelet adhesiveness using citrated plasma.

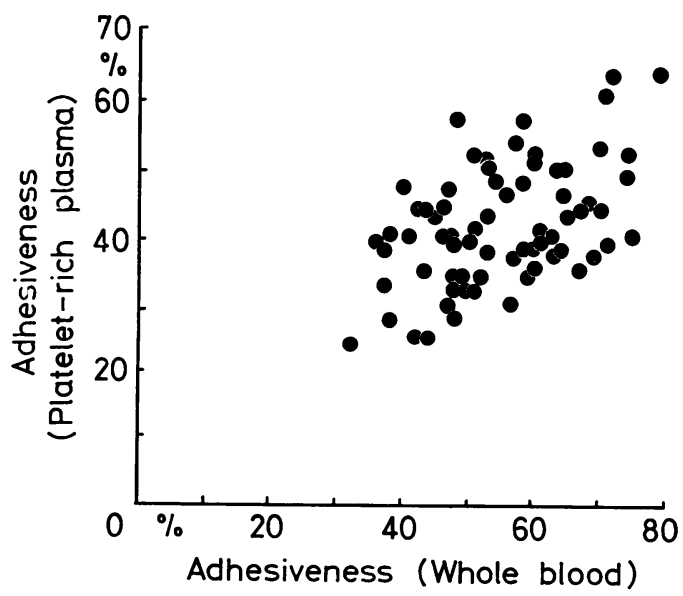

Fig. 3 Results of duplicate adhesiveness tests using whole blood and platelet-rich plasma. with a variation of $19 \%$ to $65 \%$ at one hour and $\Omega$ $30 \%$ to $64 \%$ at two hours. After standardizing our experimental conditions in the manner outlined, 70 duplicate adhesiveness tests using whole $\stackrel{\overrightarrow{0}}{\vec{O}}$ blood and platelet-rich plasma from each healthy donor were performed. The results are shown in $\overrightarrow{\overline{\vec{F}}}$ Figure 3.

The coefficient of correlation between the $\frac{\bar{\sigma}}{\sigma}$ results with the two methods is $r_{x y}=0.46$ which is $\overline{\bar{s}}$ highly significant $(P=0.001)$. However in 28 of the $\overparen{\Phi}$ 70 persons examined the results varied from the mean in opposite directions; in 18 the results ${ }^{\infty}$. were above the mean using whole blood but $\vec{\circ}$ below with citrated plasma, while in 10 the results $\overrightarrow{\vec{\omega}}$ were above the mean using citrated plasma but $\stackrel{\omega}{\omega}$ below with the whole blood method.

\section{Discussion}

In experiments on platelet adhesiveness it is important to standardize as far as possible the $z$ technique to be used and to have a large number of results on healthy persons before attempting to $\stackrel{\widehat{\rho}}{ }$ evaluate results which may be obtained in disease $\overrightarrow{0}$ processes. Although many of the factors in- $-\mathrm{D}$ fluencing the adhesiveness are known, it seems likely that others, as yet unknown, also play å part and these may come to light when the exact mechanism of platelet adhesiveness and aggre-م् gation is fully understood. The present study shows that by using two techniques of platelet adhesiveness under standardized conditions a음 good correlation in the results can be obtained. This is so, even though the normal range is fairly wide using both techniques. Hirsch, McBride and Wright (1966) found a similar correlation in the results of adhesiveness to glass using the glassoflask method of Payling Wright (1941) and passage through glass microspherules. Followingô the initial observations of Payling Wright (1942), many workers have recorded increased adhesive-은 ness after an operation using a variety of pro- $\rightarrow$ cedures, glass adhesion methods being those most frequently used, with or without, addedN ADP. Bennett (1968) has shown a discrepancy between the results with platelet-rich plasma with응 added ADP followed by glass adhesion, and glass adhesion alone using whole blood, following surgery. He does not record any observations ire healthy donors or indicate the degree of corre-s lation between the two tests before operation.? In a recent article by Sjögren, Böttiger, and $\frac{0}{0}$ Biörck (1969), four methods of platelet adhesive- $\frac{}{\Phi}$ ness were compared. They found a good corre $\frac{\rho}{9}$ lation between Hellem's and Salzman's whole blood methods but could find no significanto correlation between three whole blood methods? and Hellem's platelet-rich plasma method witho added ADP. It is suggested that the more the factors which influence the results using various? techniques can becontrolled, the greater will be the 
likelihood of individual techniques which measure the same aspect of adhesiveness to produce comparable results. In the present tests we were unable to standardize conditions in the donors, as although the blood was always taken in the morning, the food intake and type of food, the degree of exercise, and the use of tobacco or drugs were not regulated. Had this been achieved our results might have shown an even better correlation. Whether, in fact, the test using ADP plus adhesiveness and that with glass adhesion alone, measure exactly the same aspect of stickiness, is debatable, but in this study on normal donors the correlation of results suggests that it is probable. It is now intended to use these techniques in parallel in situations where increased adhesiveness is to be expected to see if closely comparable results can be obtained as have been found in healthy subjects.

We are grateful to the Sub-Committee for Clinical Research, Charing Cross Hospital for financial support.

\section{References}

Bennett, P. N. (1968). Role of erythrocytes in the acute platelet response to operation. J. clin. Path., 21, 695-697.

Bloom, A. L., and Davies, A. J. (1967). Adenosine diphosphate and the measurement of platelet adhesiveness. J. clin. Path., 20, 706-707.

Bridges, J. M., Dalby, A. M., Millar, J. H. D., and Weaver, J. A. (1965). An effect of D-glucose on platelet stickiness. Lancet, 1, 75-77.

Eastham, R. D. (1964). Rapid adhesive platelet count in whole blood. J. clin. Path., 17, 45-46.

Fyfe, T., and Hamilton, E. (1967). Effect of variation of the interval between venepuncture and measurement of platelet adhesiveness by the Payling-Wright method. Lancet, 2, 542-543.
Hellem, A. J. (1960a). The adhesiveness of human blood platele(S) in vitro. Scand. J. clin. Lab. Invest., suppl. 51, p. 12.

Hellem, A. J. (1960b). J. clin. Lab. Invest., suppl., 51, p. 12.

Hirsch, J., McBride, J. A., and Wright, H. P. (1966). PlateleP adhesiveness: a comparison of the rotating bulb and glasst bead column methods. Thrombos. Diathes. haemmorro (Stuttg.), 16, 100-104.

Ikkala, E., Myllylä, G., and Sarajas, M. S. S. (1966). Platelef: adhesiveness and ADP-induced platelet aggregation fil exercise. Ann. Med. exp. Fenn., 44, 88-92.

McBride, J. A. (1968). Platelet adhesiveness: the effect of cent fugation on the measurement of adhesiveness in platele rich plasma. J. clin. Path., 21, 397-401.

McDonald, L., and Edgill, M. (1958). Dietary restriction an coagulability of the blood in ischaemic heart-diseas? Lancet, 1, 996-998.

Murchison, L., and Fyfe, T. (1966). Lancet, 2, 182-184.

O'Brien, J. R. (1966). Platelet stickiness. Ann. Rev. Med., 1 бु 275-290.

O'Brien, J. R. (1967). Platelets: their role in haemostasis and Thrombosis. Thrombos. Diathes. haemorr. (Stuttg. suppl. 26, 185.

O'Brien, J. R., and Heywood, J. B. (1967). Some interactio between human platelets and glass: von Willebrands disease compared with normal. J. clin. Path., 20, 56-64

O'Brien, J. R. (1968). Effects of salicylates on human platelets Lancet, 1, 779-783.

O'Brien, J. R. (1969). Platelet aggregation tests and thrombosis. $\vec{\not}$ roy. Coll. Phycns Lond., 3, No. 2, 193-200.

Payling Wright, H. (1941). The adhesiveness of blood platele in normal subjects with varying concentrations of anticoagulants. J. Path. Bact., 53, 255-262.

Payling Wright, H. (1942). Changes in the adhesiveness of bloci platelets following parturition and surgical operation J. Path. Bact., 54, 461-468.

Sjögren, A., Böttiger, L. E., and Biörck, G. (1969). Platelèt adhesion: a comparison of four methods. Acta me scand., 185, 127-131. 